\title{
Biomechanical Effects of Position-Loading During Cervical Rotatory Manipulation: A Finite Element Analysis
}

\section{Huihao Wang}

Shanghai University of TCM

Hongsheng Zhan

Shanghai University of TCM

\section{Kuan Wang}

Tongji University School of Medicine

\section{Zhen Deng}

Shanghai Baoshan Hospital of Integrated Traditional Chinese and Western Medicine

Xiaofei Li

Stony Brook University, Stony Brook

\section{Zhongxiang Yu ( $\sim$ 404047854@qq.com )}

Shanghai University of TCM

\section{Research}

Keywords: Cervical spine, Facet joint degeneration, Cervical rotatory manipulation (CRM), Kinematics, Finite element analysis (FEM)

Posted Date: September 22nd, 2020

DOl: https://doi.org/10.21203/rs.3.rs-78149/v1

License: (9) This work is licensed under a Creative Commons Attribution 4.0 International License. Read Full License 


\section{Abstract}

Background: Cervical rotatory manipulation(CRM) is one of the most common treatments for neck pain in China. This study utilized a finite element method (FEM) to model the biomechanical effects of positioning during CRM in a normal sitting position.

Methods: Passive head kinematics data, including angular displacement in six directions, were recorded. These data were then imported and a finite element model constructed, then validated. Relative angular displacements, and stress on the intervertebral discs and articular surfaces were calculated in a standard symmetry model (SSM) and compared with a pathological model (PM).

Results: The results indicate that the intervertebral disc pressure (IDP) at the C2-C3, C3-C4 and C4-C5 discs subjected to CRM with position-loading were lower than the pressures experienced without positionloading. Furthermore, IDP on the target C5-C6 segment during CRM with position-loading in the PM was lower than that without position-loading, the former being close to the IDP of the C5-C6 segment during CRM without position-loading in the SSM. The left articular surface contact pressure of the target C5-C6 segment subjected to CRM towards the right without position-loading in the PM was slightly lower than that of the right articular surface experiencing leftward manipulation and lower than that predicted by the SSM. However, contact pressure of the C5-T1 segment in leftward CRM with position-loading in the PM and C6-T1 in rightward CRM with position-loading in the PM were significantly higher than the standard threshold, especially for contact pressure on the articular surfaces of C5-C6 in leftward CRM with position-loading in the PM.

Conclusions: CRM should be considered a promising strategy to mitigate neck pain in patients.

\section{Introduction}

Neck pain is among the top five chronic pain conditions in terms of prevalence. It can cause disability and consequently puts an enormous financial burden on medical resources, in addition to other direct or indirect expenses [1-3]. Cervical manipulation is commonly used for the treatment of patients with neck pain and has demonstrable effects on the function of the autonomic nervous system, sensory system, range of motion of the neck and levels of disability, in addition to its level of safety $[4,5]$.

Cervical rotatory manipulation (CRM) is recommended as a manual technique for the treatment of patients with neck pain and related disorders. There are varying aspects to the theory and practice used in Chinese manipulation academic schools [6,7]. Although a number of studies have reported that CRM is able to provide pain relief, a reduction in muscle soreness and tension for patient with neck pain, the biomechanical effects following the use of this technique with or without position-loading remain unclear for patients with neck disorders.

Biomechanical models have been used in order to understand the basic normal functions of the cervical spine and modes of dysfunction. Computational models can be developed from mathematical equations 
that incorporate the geometric and physical characteristics of the human spine and may be used to solve problems difficult to model by other means. For example, change in disc and vertebral stresses in response to graded transection of facet joints or the study of changes in endplate loading caused by disc degeneration [8] can be accomplished in this way. Following years of research and development, finite element methods (FEMs) have become a reliable approach to the study of the biomechanics of manual therapy. The authors have validated and explored the hypotheses during multiple combined loading modes and numerous boundary conditions $[9,10]$. From the results of previous research, we have simulated cervical rotatory manipulation with position-loading on the top and root of C5 respectively, concluding that the technique could significantly alter the regions of stress surrounding the cervical vertebrae, both in location and magnitude, but making no direct contribution to the reduction in intradiscal pressure [11].

Therefore, the purpose of the present study was to investigate the biomechanical effects of CRM in the sitting position with and without position-loading via finite element analysis. A cervical finite element model was established and validated in previous studies $[12,13]$.

\section{Materials And Methods}

\subsection{Data collection of skull kinematics}

A healthy Chinese male volunteer (aged 25 years, height $170 \mathrm{~cm}$, body weight $63 \mathrm{~kg}$ ) was recruited for this study. The operator was a clinical specialist (Doctor of traditional Chinese medicine) with 30 years experience dealing with cervical spine disorders using manual therapy and other non-surgical treatments.

To measure head motion during CRM relative to the thorax, a helmet was customized with 4 reflective markers (Fig. 1). Three additional reflective markers were attached to the thorax (Vicon technical markers, TMvic) on the acromion and one on the spinous process of Th2. Four additional reflective markers representing the Frankfurt plane (Vicon Frankfurt markers, FMvic) were attached to the zygomatic arch with hypoallergenic tape. During the process of manipulation, motion data were recorded using an optoelectronic system (Vicon 612: 10 cameras; Oxford, United Kingdom; sampling frequency: $100 \mathrm{~Hz}$ ). Data were filtered using a fourth order Butterworth filter with a nominal cutoff frequency of $6 \mathrm{~Hz}$.

Datasets were processed for analysis and to ascertain the principal and coupled motion components in addition to the helical axis. Motion was processed using an orientation vector method to compute angular displacements in an anatomical reference frame (origin at Th2), to define the lateral bending movement, axial rotation, and flexion extension around the $x-, y-$, and $z$-axes, respectively. Positive angular values indicated right lateral bending, left axial rotation, and extension. This method has been reported in previous manuscripts $[14,15]$.

Rightward CRM can be described in the following steps: Firstly, the head of the volunteer was placed in a neutral sitting position; Secondly, the cervical spine of the volunteer was followed by the specialist's manipulation using flexion for 15 seconds, then the head was rotated rightwards to limit position-loading 
with left lateral bending for 5 seconds; Thirdly, the specialist's left thumb was positioned at the right side of the volunteer's C5 spinous process; Fourthly, the specialist's right forearm held the volunteer's tuberculum mentale; Fifthly, the specialist simultaneously thrust his left thumb towards the C5 spinous process and raised the mandible with his right forearm; Finally, the specialist brought the volunteer's head back to neutrality using his hands. Kinematic data recorded during leftward CRM are displayed in Fig. 2.

\subsection{Data collection of cervical rotation manipulation}

In vivo loading was measured in the volunteer during CRM. Dynamometric diaphragms and a Runinsense force measurement system (Walkinsense sports, Tomorrow options, UK) were used to measure adjustment forces applied to the neck of the volunteer. In order to measure these pressures, one diaphragm was attached on the spinous process of $\mathrm{C} 5$, and the other to the right tuberculum mentale. These two positions were the principal locations of loading during treatment. Forces experienced during the adjustment step, particularly peak force, were extracted so as to calculate the moment to be applied to the FE model. This method has been described in detail in a previous study [11].

\subsection{FE modeling}

A standard C5-C6 symmetry model (SSM) was created from a validated three-dimensional (3D) non-linear FE model of an intact C5-C6 spine developed previously with transverse computed tomography (CT) images [16]. CT images were recorded at $0.625 \mathrm{~mm}$ intervals within the C5-C6 section of a healthy 30 year old male in a supine position. A pathological model (PM) of a degenerated facet joint was created from the CT images of a neck pain patient.

Facet joints play an important role in maintaining the stability of the spine when functioning in extension, flexion and rotation over a particular range. Previous studies have demonstrated that facet joint degeneration is a common cause of spinal pain $[17,18]$. It has been demonstrated that facet joint degeneration can result from abnormal motion associated with disc degeneration, in addition to arthritis, and similar to that observed in other synovial joints $[19,20]$.

Spinal facet joints have been shown to possess an abundant supply of nerves [21, 22] and it has been demonstrated that they are susceptible to arthritic and degenerative changes, inflammation, and injury, all of which can lead to a restricted range of motion and pain during movement $[23,24]$.

In the PM, during $\mathrm{C} 5$ vertebral dextrorotation i.e. the $\mathrm{C} 5$ spinous process undergoing levorotation, the C5C6 left articular facet moved forward $15^{\circ}$ relative to the right articular facet. In addition, the C5-C6 left capsular ligament exhibited linear stiffness while the right side displayed nonlinear stiffness. Other characteristics of the PM were the same as those of the SSM.

\subsection{FE analysis}

The bilateral boundary conditions of the SSM and PM were identical. All nodes on the lower surfaces of C6 were fixed rigidly. Simulated rightward CRM only was performed on the SSM because of its symmetrical characteristics, the results on both sides considered equivalent in this model. Complete 
simulated CRM was performed for three different loading conditions. The specific details of the simulation are displayed in Fig. 3:

(1) Pure head loading: A 50-N preload was applied to the superior faces of $\mathrm{C} 5$ to simulate the weight of the head. This loading simulated the mechanical state of the cervical spine in the pre-manipulation position.

(2) Adjustment period: Angular displacement (AD) data of head kinematics captured during CRM were applied on the models, consisting of four phases including flexion, rotation, lateral bending to limit position and thrust force. The AD data in three planes at the end of each time point were recorded for each phase. (1) and (2) represent the CRM procedure without simulation of position-loading.

(3) Position loading: A 68-N force was applied perpendicular to the axis of the $\mathrm{C} 5$ spinous process with deviation to the right in the PM. For comparison, reverse direction parameters were also applied to the models, which were recorded in the AD data as head kinematics and thrust force.

In summary, three conditions were simulated in the present study, unilateral CRM without position-loading in the SSM, bilateral CRM without position-loading in the PM and bilateral CRM with position-loading in the PM. FE simulations were performed in static mode using ABAQUS standard solver software.

\section{Results}

\subsection{Effect on angular displacement}

Relative angular displacement during rightward CRM without position-loading in the PM was significantly higher than during leftward manipulation at the C5-C6 level, and both significantly greater than the relative angular displacement after CRM without position-loading on the SSM. Furthermore, relative angular displacements during CRM with position-loading at the C5-C6 and C6-C7 levels were apparently greater than those during CRM without position-loading. However, relative angular displacements during CRM with position-loading at the C2-C3, C3-C4 and C4-C5 levels were lower than those induced by CRM without position-loading. Detailed results of the relative angular displacement in each cervical segment during CRM are listed in Table 1. 
Table 1

The relative angular displacement in each cervical segment during CRM $\left(^{\circ}\right)$

\begin{tabular}{|c|c|c|c|c|c|}
\hline Unit & $\begin{array}{l}\text { SSM - CRM } \\
\text { without } \\
\text { position }\end{array}$ & $\begin{array}{l}\text { PM - CRM } \\
\text { without position - } \\
\text { Leftward }\end{array}$ & $\begin{array}{l}\text { PM - CRM without } \\
\text { position - } \\
\text { Rightward }\end{array}$ & $\begin{array}{l}\text { PM - CRM with } \\
\text { position - } \\
\text { Leftward }\end{array}$ & $\begin{array}{l}\text { PM - CRM with } \\
\text { position - } \\
\text { Rightward }\end{array}$ \\
\hline $\begin{array}{l}\text { C2 - } \\
\text { C3 }\end{array}$ & 5.9144 & 5.8365 & 5.8551 & 5.4733 & 5.4869 \\
\hline $\begin{array}{l}\text { C3 - } \\
\text { C4 }\end{array}$ & 6.2643 & 6.192 & 6.1788 & 5.8567 & 5.8286 \\
\hline $\begin{array}{l}\text { C4 - } \\
\text { C5 }\end{array}$ & 6.5659 & 6.4562 & 6.6416 & 6.1357 & 6.3078 \\
\hline $\begin{array}{l}\text { C5 - } \\
\text { C6 }\end{array}$ & 6.9663 & 7.166 & 7.5859 & 8.9201 & 9.5568 \\
\hline $\begin{array}{l}\text { C6 - } \\
\text { C7 }\end{array}$ & 3.916 & 3.8685 & 3.9871 & 4.3882 & 4.4864 \\
\hline $\begin{array}{l}\text { C7 - } \\
\text { T1 }\end{array}$ & 4.2139 & 4.1786 & 4.2078 & 4.378 & 4.3745 \\
\hline
\end{tabular}

\subsection{Effect on intervertebral discs}

The proportion of change in IDP during the impulse and neutral phases during CRM with or without position-loading in the SSM and PM are displayed in Table 2. From the overall trend, the lower intervertebral disc segments experienced higher relative change in IDP. As expected, the IDP was different in the SSM and PM. Furthermore, the percentage change in IDP at the C5-C6 level during bilateral CRM without position-loading in the PM were clearly higher than the change in CRM without position-loading on the SSM and bilateral CRM with position-loading in the PM. However, at the C4-T1 level, the percentage change in IDP was slightly lower during rightward CRM without position-loading in the PM than during leftward manipulation. In comparison, the tendency for increased IDP during CRM with position-loading in the PM at the $\mathrm{C} 2-\mathrm{C} 6$ levels was lower than during CRM without position-loading in the PM, except for increased IDP that was observed at the C7-T1 segment. 
Table 2

Percentage change in IDP during the impulse and neutral phases (\%)

\begin{tabular}{|c|c|c|c|c|c|}
\hline Unit & $\begin{array}{l}\text { SSM- CRM } \\
\text { without } \\
\text { position }\end{array}$ & $\begin{array}{l}\text { PM - CRM } \\
\text { without position - } \\
\text { Leftward }\end{array}$ & $\begin{array}{l}\text { PM - CRM without } \\
\text { position - } \\
\text { Rightward }\end{array}$ & $\begin{array}{l}\text { PM - CRM with } \\
\text { position - } \\
\text { Leftward }\end{array}$ & $\begin{array}{l}\text { PM - CRM with } \\
\text { position - } \\
\text { Rightward }\end{array}$ \\
\hline $\begin{array}{l}\mathrm{C} 2- \\
\mathrm{C} 3\end{array}$ & 193.9286 & 195.9035 & 198.6434 & 176.9757 & 179.4032 \\
\hline $\begin{array}{l}\text { C3 - } \\
\text { C } 4\end{array}$ & 239.927 & 242.2993 & 245.5165 & 221.6557 & 224.4918 \\
\hline $\begin{array}{l}\mathrm{C} 4- \\
\mathrm{C} 5\end{array}$ & 270.3024 & 273.1736 & 274.8781 & 246.7105 & 247.6626 \\
\hline $\begin{array}{l}\text { C5 - } \\
\text { C6 }\end{array}$ & 238.4362 & 277.3668 & 283.0365 & 256.4814 & 253.5489 \\
\hline $\begin{array}{l}\text { C6 - } \\
\text { C } 7\end{array}$ & 313.7061 & 328.4763 & 308.6191 & 346.5192 & 324.4603 \\
\hline $\begin{array}{l}\text { C7 - } \\
\text { T1 }\end{array}$ & 359.9651 & 378.3925 & 360.0371 & 501.8886 & 483.0546 \\
\hline
\end{tabular}

Figure 4 displays the distribution of IDP during the impulse phase. Stress concentration was located at the lateral margins of the fibrous annulus of the intervertebral discs on the rotatory side of the cervical spine. Nevertheless, there was no significant difference in IDP distribution for the five different experiments.

\subsection{Effect on articular surface contact pressure}

During the impulse phase, articular surface contact pressure only occurred on the opposite side to that of the rotation. Table 3 presents the articular surface contact pressure observed in the five experimental conditions. In the present study, the articular surface contact pressure of the C2-T1 levels during CRM without position-loading in the PM was established as $100 \%$ of the standard threshold. Taken together, the contact pressure of the bilateral CRM without position-loading in the PM was close to the standard threshold. However, contact pressure at the C5-T1 segment during leftward CRM with position-loading in the PM and at the C6-T1 segment during rightward CRM with position-loading in the PM were significantly higher than the standard threshold, especially during articular surface contact pressure of C5-C6 during leftward CRM with position-loading in the PM. In comparison, contact pressure of the C5-C6 during the other three experimental conditions was lower than the standard threshold. 
Table 3

Articular surface contact pressure under the five experimental conditions (\%)

\begin{tabular}{|c|c|c|c|c|c|}
\hline Unit & $\begin{array}{l}\text { SSM- CRM } \\
\text { without } \\
\text { position }\end{array}$ & $\begin{array}{l}\text { PM - CRM } \\
\text { without position - } \\
\text { Leftward }\end{array}$ & $\begin{array}{l}\text { PM - CRM without } \\
\text { position - } \\
\text { Rightward }\end{array}$ & $\begin{array}{l}\text { PM - CRM with } \\
\text { position - } \\
\text { Leftward }\end{array}$ & $\begin{array}{l}\text { PM - CRM with } \\
\text { position - } \\
\text { Rightward }\end{array}$ \\
\hline $\begin{array}{l}\text { C2 - } \\
\text { C3 }\end{array}$ & 100 & 101.7873 & 95.94782 & 95.95869 & 89.47009 \\
\hline $\begin{array}{l}\text { C3 - } \\
\text { C4 }\end{array}$ & 100 & 101.9763 & 95.91953 & 96.25877 & 89.45995 \\
\hline $\begin{array}{l}\text { C4 - } \\
\text { C5 }\end{array}$ & 100 & 103.3909 & 94.03224 & 98.64505 & 88.60453 \\
\hline $\begin{array}{l}\text { C5 - } \\
\text { C6 }\end{array}$ & 100 & 96.05947 & 72.71907 & 125.928 & 95.72908 \\
\hline $\begin{array}{l}\text { C6 - } \\
\text { C7 }\end{array}$ & 100 & 100.3151 & 95.96001 & 138.7231 & 133.3812 \\
\hline $\begin{array}{l}\mathrm{C} 7 \\
\overline{\mathrm{T}} 1\end{array}$ & 100 & 101.2132 & 94.23009 & 158.4397 & 150.2085 \\
\hline
\end{tabular}

\section{Discussion}

Spinal manipulative therapy (SMT) is a widely used manual treatment, but it has not been currently possible to provide overall conclusions about the safety of SMT [25]. Stress and strain on the neck during SMT have been found to vary greatly among clinicians and the locations of treatment on the spine [26]. With the continuous intensification of multidisciplinary and interdisciplinary research, the combination of biomechanics on the human musculoskeletal system and manual therapy has gradually become a focus of attention in the field of biomechanics in sports and rehabilitation medicine in which the methodology and technology of traditional Chinese medicine (TCM) is being investigated $[27,28]$. In the present study, the biomechanical effects of three conditions were simulated and analyzed during CRM both with and without position-loading in an SSM and PM.

(1) Effect on angular displacement

The results indicated that there was no apparent distinction between the SSM and PM during CRM both with and without position-loading for relative angular displacement. However, the C5-C6 level in the PM experienced large angular displacement during CRM with position-loading due to the thumb thrust force on the left side of the C5 spinous process, corresponding to the experience of clinical therapy. In addition, angular displacement of segments above the C5-C6 level was relatively lower than during CRM with position-loading. However, angular displacement of the segments below the target level was relatively higher. The C5-C6 segment is located in the middle and lower part of the cervical spine, close to the C7-T1 segment. Because of the limited degrees of freedom of the lower surface of T1 in each direction, larger 
angular displacements could be generated below the target level during loading of the thumb thrust force on the $\mathrm{C} 5$ spinous process. Conversely, the $\mathrm{C} 0-\mathrm{C} 2$ segment of the cervical spine experienced a large horizontal rotation, which to a certain degree compensated for the coupling force of the target segment.

\section{(2) Effect on intervertebral discs}

During CRM with position-loading, the IDP at C2-C3, C3-C4 and C4-C5 was lower than during CRM without position-loading, consistent with changes in relative angular displacement at the same levels.

Furthermore, IDP of the target C5-C6 segment during CRM with position-loading on the PM was lower than that during CRM without position-loading, the former being close to the magnitude of IDP in the C5C6 segment during CRM without position-loading in the SSM. It is, however, worth noting that the IDP of the C7-T1 segment significantly increased during CRM with position-loading. This is probably due to the limited degrees of freedom of the lower surface of $\mathrm{T} 1$ in each direction. Furthermore, these results indicate that stress concentrated at the lateral margins of the fibrous annulus of the intervertebral discs on the rotatory side of the cervical spine during CRM. Previous studies confirm that stress on the disc and the tissues between the cervical nerves, spinal cord and other structures increase following degeneration of the intervertebral discs. [29] Therefore, we believe that it is safer for patients with intervertebral disc degeneration to undergo CRM with position-loading in order that the increase in IDP is limited.

\section{(3) Effect on articular surface contact pressure}

For comparison, the articular surface contact pressure of the C2-T1 levels during CRM without positionloading in the PM was established as $100 \%$ of the standard threshold. Results demonstrated that the left articular surface contact pressure of the target C5-C6 segment during rightward CRM without positionloading in the PM was slightly lower than right articular surface contact pressure during leftward manipulation and lower than in the SSM. However, contact pressure at the C5-T1 during leftward CRM with position-loading in the PM and at C6-T1 during rightward CRM with position-loading on the PM were significantly higher than the standard threshold, especially that of the articular surface contact pressure at C5-C6 during leftward CRM with position-loading in the PM. In comparison, contact pressure at C5-C6 during the other three experimental conditions was lower than the standard threshold. The results agree with the characteristics of the pathological mechanism, indicating that the C5-C6 left capsular ligament exhibited linear stiffness, while the stiffness on the right side was nonlinear. Therefore, it is more appropriate for the target C5-C6 pathological segment and segments below during CRM with positionloading to use the same static loading. However, future studies should focus on the rapid increase in articular surface contact pressure and whether this would result in iatrogenic damage or other adverse effects.

There are several limitations to the present study which should be noted. Firstly, the simulations were performed under idealized conditions that did not consider muscle forces in the neck. Because of the significant non-linear material properties of muscles, characteristics of the muscular self-reflex system and control by the brain of the muscles, simulating the function of the muscles was difficult using FE analysis at the present stage. The present study assumed that the volunteer had undergone CRM with 
complete muscle relaxation with no resistance. Secondly, FE static analysis was used to ensure higher calculation accuracy. Nevertheless, time was not taken into account in this study, even though viscoelasticity of the ligaments is time-dependent. Therefore, FE dynamic analysis of viscoelasticity of the ligaments could be further analyzed to study the biomechanical state of the cervical spine when restored to neutrality following the impulse phase. Future investigations might require consideration of these factors and provide a deeper understanding of the biomechanics of CRM in a sitting position with and without position-loading via finite element analysis and represent a more useful reference for clinical guidance.

\section{Conclusions}

The present study demonstrated that CRM with position-loading was safer for patients with cervical disc degeneration, with stress distributed more evenly in a target pathological C5-C6 segment. Additionally, a PM was established where the C5-C6 left articular facet moved forward $15^{\circ}$ more than the right articular facet and the C5-C6 left capsular ligament had developed linear stiffness. During offside (right) manipulation, articular surface contact pressure of the target pathological C5-C6 segment did not reach the standard threshold. Clinicians might consider omitting that step to avoid creating potential lesions.

\section{Declarations}

\section{Ethical Approval and Consent to participate}

The China Ethics Committee of Registering Clinical Trials' Ethical Review Board approved this study (No. ChiECRCT-20150076), and the procedures followed were in accordance with the Helsinki Declaration of 1975 , as revised in 2000 . Written informed consent will be obtained from all participants prior to enrolment in the study.

\section{Consent for publication}

Consent for publication was obtained from all participants.

\section{Availability of supporting data}

All data generated or analysed during this study are included in this published article.

\section{Competing interests}

The authors declare that they have no competing interests.

\section{Funding}

This research was supported the National Natural Science Foundation of China (No. 81973871, 81804114) and the clinical research project of Shanghai University of Traditional Chinese Medicine (No. 
18LK038).

\section{Authors' contributions}

$\mathrm{HW}$ and $\mathrm{ZY}$ conceived and designed the study. $\mathrm{HZ}$ and $\mathrm{KW}$ obtained the data and conducted data formatting. ZD and XL helped plot the results. All authors helped interpret the findings and wrote the draft of the manuscript. All authors have had full access to all of the data in the study and can take responsibility for its integrity and the accuracy of the data analysis.

\section{Acknowledgements}

We thank Prof. Yongfang Zhao, Prof. Jian Pang and the other crew of Shi's Center of Orthopedics and Traumatology, Shuguang Hospital Affiliated to Shanghai University of TCM for the help in the guidance of scientific ideas to experimental design, and Dr. M Zhang and Dr. W Tan for the radiological technical support.

\section{References}

1. A. Popescu, H. Lee, "Neck Pain and Lower Back Pain," Med Clin North Am, vol. 104, no. 2, pp. 279292, 2020.

2. S. P. Cohen and W. M. Hooten, " Advances in the diagnosis and management of neck pain," $B M J$, vol. j3221, 2017.

3. E. K. Hansson and T. H. Hansson, "The costs for persons sick-listed more than one month because of low back or neck problemsA two-year prospective study of Swedish patients," European Spine Journal, vol. 14, no. 4, pp. 337-345, 2005.

4. P. R. Blanpied, A. R. Gross, J.M. Elliott, L. D. Laurie, C. Derek, M. W. David, S. Cheryl and K. R. Eric, “Neck Pain: Revision 2017," Journal of Orthopaedic \& Sports Physical Therapy, vol. 47, no. 7, pp. A1A83, 2017.

5. P. Côté, J. J. Wong, D. Sutton, et al, “ Management of neck pain and associated disorders: A clinical practice guideline from the Ontario Protocol for Traffic Injury Management (OPTIMa) Collaboration," European Spine Journal, vol. 25, no. 7, pp. 2000-2022, 2016.

6. L. Zhu, M. Feng, X. Yin, S. Wang and J. Yu, " Kinematics Analysis of Cervical Rotation-Traction Manipulation Measured by a Motion Capture System," Evidence-Based Complementary and Alternative Medicine, vol. 2017, pp. 1-6, 2017

7. H. L. Jian, S. Tong, R. C. K. Chung and T. T. W. Chiua, "The effectiveness of Long's manipulation on patients with chronic mechanical neck pain: A randomized controlled trial," Man Ther, vol. 18, no. 4, pp. 308-315, 2013.

8. M. M. Panjabi, “ Cervical Spine Models for Biomechanical Research," Spine, vol. 23, no. 24, pp. 26842699, 1998. 
9. L. Wan, J. Chen and Y. K. Li, " Three-dimensional anatomical position of rotatory center in cervical rotatory and local manipulation," Journal of Southern Medical University, vol. 28, no. 4, pp. 548-550, 2008

10. H. W. Ng and E. C. Teo, "Nonlinear Finite-Element Analysis of the Lower Cervical Spine (C4-C6) Under Axial Loading," Journal of Spinal Disorders, vol. 14, no. 3, pp. 201-210, 2001.

11. Z. Deng, K. Wang , H. Wang, T. Lan and W. Niu, " A finite element study of traditional Chinese cervical manipulation," European Spine Journal, vol. 26, no. 7, pp. 1-10, 2017.

12. K. Wang , H. Wang, Z. Deng, Z. Li, H. Zhan and W.Niu, "Cervical traction therapy with and without neck support: A finite element analysis," Musculoskeletal Science and Practice, vol. 28, pp. 1-9, 2017.

13. H. Wang, K. Wang, Z. Deng, X. Li , Y. Qin, H. Zhan and W. Niu, " Effects of facet joint degeneration on stress alterations in cervical spine C5-C6: A finite element analysis," Mathematical Biosciences and Engineering, vol. 16, no. 6, pp. 7447-7457, 2019.

14. P. M. Dugailly, S. Sobczak, V. Sholukha, S. V. S. Jan and Rooze, M, “ In vitro 3D-kinematics of the upper cervical spine: Helical axis and simulation for axial rotation and Xexion extension," Anatomia Clinica, vol. 32, no. 2, pp. 141-151, 2009.

15. Dugailly P M, Sobczak S, Van Geyt B, et al, " Head-trunk kinematics during high-velocity-lowamplitude manipulation of the cervical spine in asymptomatic subjects: helical axis computation and anatomic motion modeling," Journal of Manipulative \& Physiological Therapeutics, vol. 38, no. 6, pp. 416-424, 2015.

16. K. Wang, Z. Deng, H. Wang, Z. Li, H. Zhan and W. Niu, "Influence of variations in stiffness of cervical ligaments on C5-C6 segment," Journal of the Mechanical Behavior of Biomedical Materials, vol. 72, pp. 129-137, 2017.

17. L. Manchikanti, A. D. Kaye, M. V. Boswell, S. Bakshi, and J. A. Hirsch, " A Systematic Review and Best Evidence Synthesis of the Effectiveness of Therapeutic Facet Joint Interventions in Managing Chronic Spinal Pain," Pain Physician, vol. 18, no. 4, pp. E535-E582, 2015.

18. M. V. Boswell, L. Manchikanti, A. D. Kaye, S. Bakshi and J. A.Hirsch, " A Best-Evidence Systematic Appraisal of the Diagnostic Accuracy and Utility of Facet (Zygapophysial) Joint Injections in Chronic Spinal Pain," Pain Physician, vol. 18, no. 4, pp. E497-E533, 2015.

19. J. L. Bykowski and W. H. W. Wong, "Role of Facet Joints in Spine Pain and Image-Guided Treatment: A Review," American Journal of Neuroradiology, vol. 33, no. 8, pp. 1419-1426, 2012.

20. L. Kalichman, L. Li, D. H. Kim, et al, " Facet Joint Osteoarthritis and Low Back Pain in the CommunityBased Population," Spine, vol. 33, no. 23, pp. 2560-2565, 2008.

21. S. Kallakuri, Y. Li, C. Chen and J. M.Cavanaugh, " Innervation of cervical ventral facet joint capsule: Histological evidence," World Journal of Orthopaedics, vol. 3, no. 2, pp. 10-14, 2012.

22. A. Siegenthaler, J. Schliessbach, M. Curatolo, et al, "Ultrasound anatomy of the nerves supplying the cervical zygapophyseal joints," Regional Anesthesia and Pain Medicine, vol. 36, no. 6, pp. 606-610, 2011. 
23. A. C. Gellhorn, J. N. Katz and P. Suri, "Osteoarthritis of the spine: the facet joints," Nature Reviews Rheumatology, vol. 9, no. 4, pp. 216-224, 2012.

24. E. Tessitore, G. Molliqaj, B. Schatlo and K.Schaller, "Clinical evaluation and surgical decision making for patients with lumbar discogenic pain and facet syndrome," European Journal of Radiology, vol. 84, no. 5, pp. 765-770, 2015.

25. S. M. Nielsen, S. Tarp, R. Christensen, H. Bliddal, L. Klokker and M. Henriksen, “ The risk associated with spinal manipulation: an overview of reviews," Systematic Reviews, vol. 6, no. 1, pp. 64, 2017.

26. W. Herzog, "The biomechanics of spinal manipulation," J Bodyw Mov Ther, vol. 14, no. 3, pp. 280286, 2010.

27. L. Li, T. Shen and Y. K. Li, " A Finite Element Analysis of Stress Distribution and Disk Displacement in Response to Lumbar Rotation Manipulation in the Sitting and Side-Lying Positions," Journal of Manipulative \& Physiological Therapeutics, vol. 40, no. 8, pp. 580-586, 2017.

28. J. F. Chen, X. N. Shu, S. J. Tang, Y. Wu and Y. P. Zhang, "Influence of lumbar disc degeneration on the efficacy of lumbar fixed-point rotation manipulation in sitting position: a finite element study," Journal of Acupuncture and Tuina Science, vol. 14, no. 4, pp. 295-299, 2016.

29. C. Lunghi, P. Tozzi, G. Fusco, "The biomechanical model in manual therapy: is there an ongoing crisis or just the need to revise the underlying concept and application? ," Journal of Bodywork and Movement Therapies, vol. 20, no. 4, pp. 784-799, 2016.

\section{Figures}
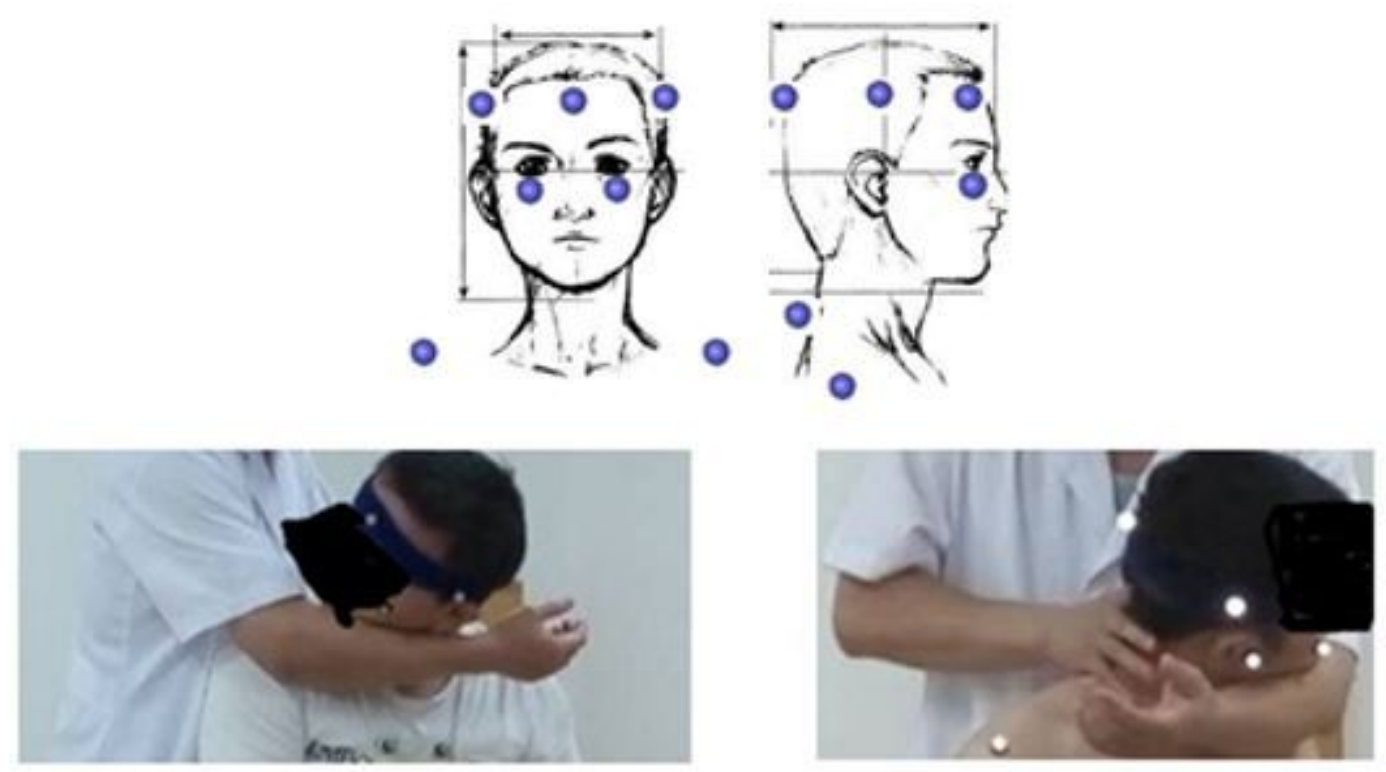

\section{Figure 1}

Location of Marker Points for CRM Motion Capture 


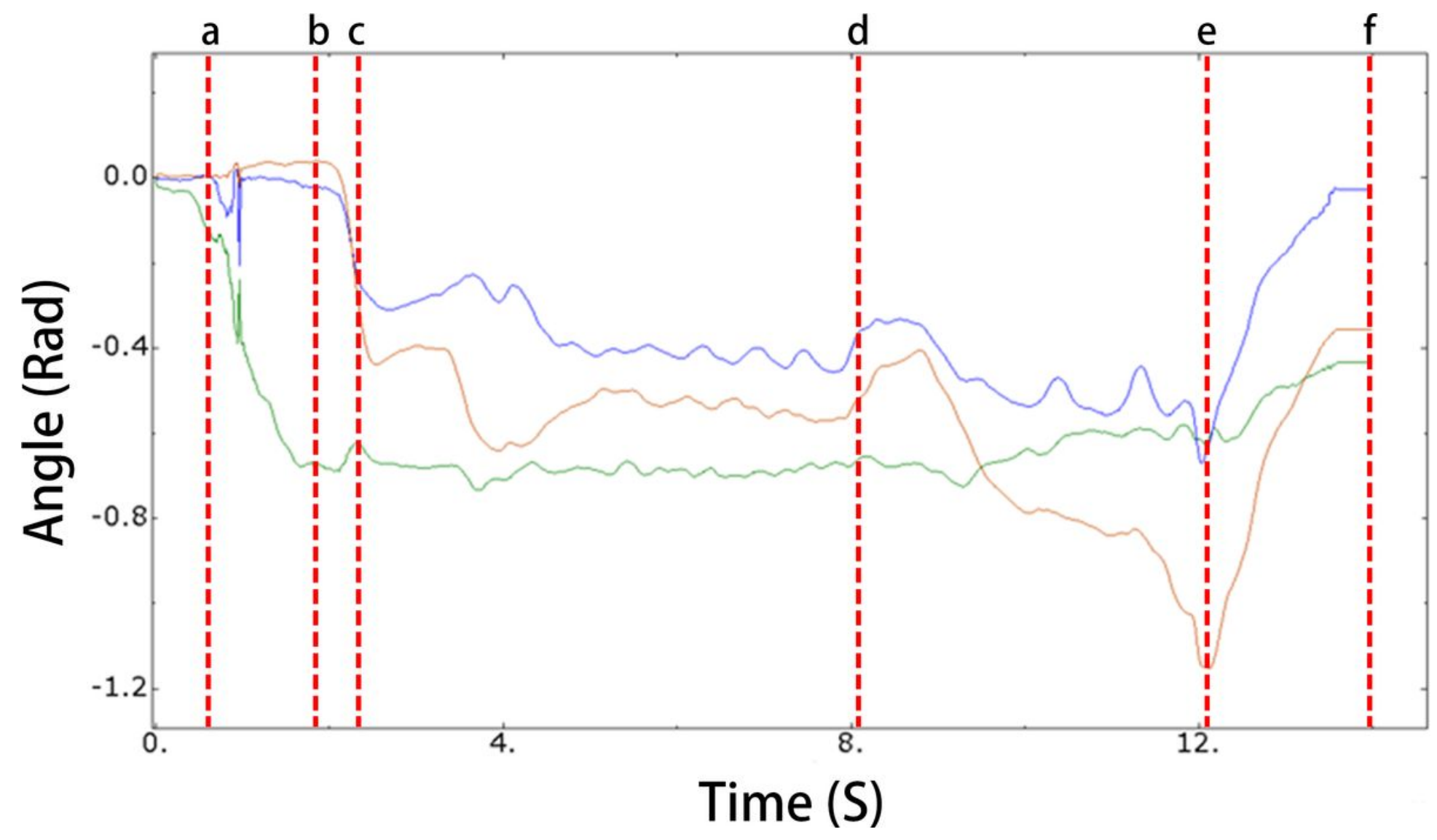

Figure 2

Kinematic readings of the head during CRM at the $\mathrm{C} 5$ level on the left side. Angular displacement in lateral bending (x-axis, anteroposterior, green), axial rotation ( $y$-axis, vertical, red), and FE (z-axis, transversal, blue). Three phases of CRM: Premanipulation phase, including flexion (ab), rotation and lateral bending (bc) and limited position (cd); Impulse phase (de); Postmanipulation phase (ef). Negative values correspond to left lateral bending, right axial rotation, and flexion.

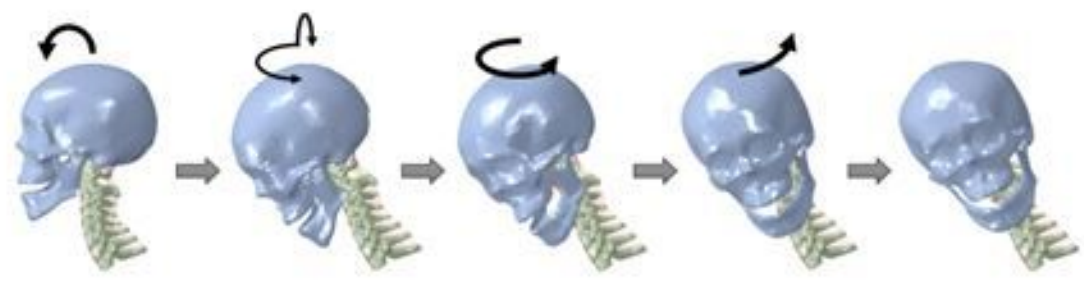

a.

Flexion

Rotation and lateral Limited Impulse

bending position phase

Figure 3 
Steps of the loading during CRM. a. CRM without position-loading b. CRM with position-loading * The red arrow indicates the position of the thrust force of the specilist's thumb
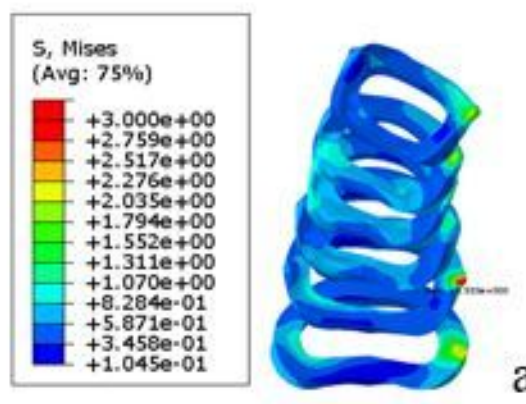

a.

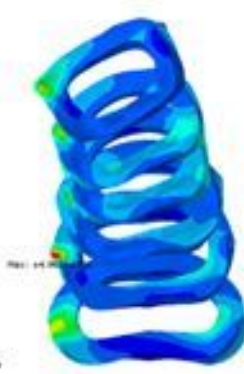

b.

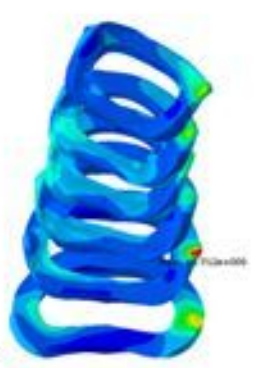

c.
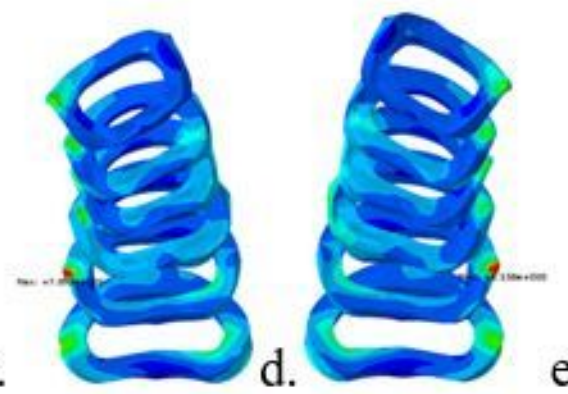

\section{Figure 4}

von Mises stress (MPa) distribution for five experimenal conditions. a. SSM- CRM without positionloading; b. PM - CRM without position-loading - Leftward; c. PM - CRM without position-loading Rightward; d. PM - CRM with position-loading - Leftward; e. PM - CRM with position-loading - Rightward 